\title{
A PUBLIC FABRIC DATABASE FOR DEFECT DETECTION METHODS AND RESULTS
}

\author{
Javier Silvestre-Blanes ${ }^{1 \star}$, Teresa Albero-Albero' ${ }^{1}$ Ignacio Miralles ${ }^{2}$, Rubén Pérez-Llorens ${ }^{1}$, Jorge Moreno ${ }^{2}$ \\ ${ }^{1}$ ITI, DISCA, Universitat Politècnica de València, UPV, València, Spain \\ ${ }^{2}$ AITEX, Plaza Emilio Sala, 1, 03801 Alcoy, Spain \\ E-mail: jsilves@disca.upv.es
}

\begin{abstract}
:
The use of image processing for the detection and classification of defects has been a reality for some time in science and industry. New methods are continually being presented to improve every aspect of this process. However, these new approaches are applied to a small, private collection of images, which makes a real comparative study of these methods very difficult. The objective of this paper was to compile a public annotated benchmark, that is, an extensive set of images with and without defects, and make these public, to enable the direct comparison of detection and classification methods. Moreover, different methods are reviewed and one of these is applied to the set of images; the results of which are also presented in this paper.
\end{abstract}

\section{Keywords:}

Fabric inspection, defect classification, machine vision system

\section{Introduction}

Automation in inspection processes within the textile industry is an area which has been analyzed since the mid-1990s. This need, in industry in general, and in particular in the textile industry [1, 2], has been widely studied. Defect detection in this sector is an important factor in reducing costs, in terms of time, and therefore in customer satisfaction.

However, this is not a simple task, and considering the difficulties involved in textile defect detection, several methods have been presented over the past 20 years [3]. However, a comparison of results of different authors is difficult, if not impossible, due to the lack of information on the images used, the fact that the types of defect differ considerably, and the resolution is not the same. In addition, as mentioned in Refs. [4] and [5], due to the huge number of fabric defect detection algorithms and techniques, an effective comparison between fabric defect detection methods would be extremely significant, but most studies use different databases, different imaging systems, and different parameters. This means that the lack of a suitable public annotated benchmark makes it difficult for researchers to evaluate, in a quantitative way, the advantages of their algorithms over existing ones, and as such this lack represents a serious limitation on the development of effective and implementable algorithms in some fields, for example, in road lane detection [6], and which also happens in the textile industry. For this reason, it is important to have a public database that can be used by different authors for their studies and comparisons, since in many cases, as mentioned in Ref. [4], in the majority of the studies "the authors create their own databases by obtaining the images from factory environments or by bringing them to the laboratory and the database is created with proper lighting settings. Therefore, the reliability and validity of the methods is far from objectivity."

The aim of this paper was to compile a public annotated database of plain fabrics (uniform fabric textures), with and without defects, so that an accurate comparison of the different methods currently available as well as any future proposals is possible. Thus, the advantages of each method can be adequately appraised using this database.

In the following section, different works carried out on the detection of defects in fabrics are analyzed, analyzing the privacy of the databases used, the quality, and the defects evaluated. The remaining sections present a review of the detection methods applied, with particular focus on the use of Gabor filters. The database (www.aitex.es/afid) is also presented, and finally the preliminary results obtained using the Gabor filter are given. With these data, researchers will be able to easily compare any newly proposed methods.

\section{Related work and textile databases}

To carry out the research work in the field of detection and classification of defects (Table 1), it is important to have a representative collection of samples, with and without defects, available that allow the results of each method used to be developed and evaluated. Table 2 summarizes the characteristics of previous work done in methods for fabric defect detection in terms of availability of images used, their properties, and type of defect and method of detection used 
in each paper. The meanings of the acronyms used are given below Table 2 to help with understanding. We wanted to summarize in the clearest possible way the characteristics of the images and databases used in the previous works mentioned. In many papers, the images used are private and little information is provided about resolution or other capture properties. This makes it impossible to verify the results and present new methods, which improve results, as it is not possible to make a comparison of results using the same information [4, 5]. Another clear drawback in the papers covered in Table 2 is that the set of images is too small to generate acceptable results that have a general application. Regarding the catalog of defects, there is not excessive uniformity in the defects that are included in these studies.

Table 1 summarizes some fabric defect types, and they are described in Refs. [7-9]. The defects available in our database are marked with $\left(^{*}\right)$. They are defects captured in a factory by a real system, after 6 months of reading. These are the most usual, and the others are more sporadic, although this may vary from factory to factory. It should be noted that 12 defects may appear to be few compared to 61 , but it is the database that contains the greatest number of defects, taking into account the previous works evaluated in Table 2.

To analyze the more generic aspects, there are many different types of image databases used by researchers. For example, there is a segmentation database and benchmark published on the Berkeley Computer Vision Group website [42], used in Ref.
[43] to work on the problem of contour detection and image segmentation or in Ref. [44] to present a new algorithm for image segmentation, Tensor-Based Image Segmentation Algorithm (TBISA). Moreover, several papers such as Refs. [45-47] have used well-known texture databases for their studies, and these include Brodatz [48] and VisTex [49], used for the detection of defects in Ref. [50] too. Other, less well-known databases are KTH-TIPS [51] and CUReT (Columbia-Utrecht Reflectance and Texture) [52]. The database of patterned fabrics used in Ref. [53] was provided by the University of Hong Kong. This database is not public. It is composed of a variable number of images as the database continues to grow over time, for example, 25 fabric images containing five types of defects in Ref. [15]; 30 defective images and 30 defect-free images in Ref. [54]; and 106 samples, 50 defect-free, and 56 defective in Ref. [55]. However, although the analysis of textures is a relevant aspect for textiles, and the techniques developed may have some application in this area, these works are not considered in this present paper as they do not focus on textiles and do not have images with defects available.

The textile databases mentioned in Table 2 have been used by a wide range of authors. The PARVIS database [18] is private, without public access. It contains two kinds of textiles with 1117 elements. The Textile Texture-Database (TILDA) [19] was developed within the framework of the working group Texture Analysis of the DFG's (Deutsche Forschungsgemeinschaft) in the Technische Universitt Hamburg in 1995. It has eight representative textile types. For each of the above classes, 50

Table 1. Fabric defect types

\begin{tabular}{|c|c|c|c|c|c|}
\hline Defect type & Defect number & Defect type & Defect number & Defect type & Defect number \\
\hline Floats & 1 & Cut selvage* & 22 & Net multiples & 43 \\
\hline Broken end* & 2 & Crease* & 23 & Loom fly & 44 \\
\hline Oil stains & 3 & Warp float & 24 & Missing draw & 45 \\
\hline Slubs & 4 & Warp Ball* & 25 & Missing weft & 46 \\
\hline Miss end & 5 & Foreign fiber & 26 & Kink & 47 \\
\hline Broken yarn* & 6 & Knots* & 27 & Unrelated corpus & 48 \\
\hline Miss pick & 7 & Harness breakdown & 28 & Burl & 49 \\
\hline Spot & 8 & Contamination* & 29 & Colorfly & 50 \\
\hline Big knot & 9 & Nep* & 30 & Broken needle & 51 \\
\hline Broken pick* & 10 & Water damage & 31 & Barre & 52 \\
\hline End out & 11 & Thick bar & 32 & Dropped stitch & 53 \\
\hline Lines & 12 & Coarse end & 33 & Warp lacking & 54 \\
\hline Fault yarns & 13 & Coarse filling & 34 & Open reed & 55 \\
\hline Wrong draw & 14 & Knees & 35 & Soiled end & 56 \\
\hline Dirty yarn & 15 & Weft crack* & 36 & Sloughed filling & 57 \\
\hline Weft curling* & 16 & Ripped & 37 & Gout & 58 \\
\hline Double weft & 17 & Double yarn & 38 & Knot with halos & 59 \\
\hline Trip warp & 18 & Miss yarn & 39 & Thick node & 60 \\
\hline Fuzzy ball* & 19 & Broken fabric & 40 & Holes & 61 \\
\hline Slack end & 20 & Roving & 41 & & \\
\hline Thin bar & 21 & Thin place & 42 & No defect & 00 \\
\hline
\end{tabular}

*Defects available in our database. 
Table 2. Data from other related works

\begin{tabular}{|c|c|c|c|c|}
\hline Paper & $\begin{array}{l}\text { Database } \\
\text { info }\end{array}$ & $\begin{array}{c}\text { Characteristics of } \\
\text { images }\end{array}$ & Fabric defects & Methods \\
\hline$[10]$ & $\mathrm{PC}$ & noi: 3700,2000 ' 512 & $4,7,11$ & Correlation \\
\hline [11] & $P$ & $\begin{array}{l}\text { ndi: } 42, \text { nwi: } 42 \\
256 \times 256\end{array}$ & $\begin{array}{l}\text { Seven, and other seven fabric } \\
\text { defects not specified }\end{array}$ & Wavelet packets \\
\hline [12] & $\mathrm{P}$ & $\begin{array}{c}\text { ndi: } 45, \text { nwi: } 8 \\
128 \times 128\end{array}$ & $3,12,15,61$ & WT, GLCM \\
\hline [13] & PC & noi: 12 & 3,13 & Mallat WT \\
\hline$[14]$ & PUB & $256 \times 256$ & $2,6,7,9,14,15,17,18$ & FIR filters \\
\hline [15] & $\mathrm{P}$ & noi: $25,256 \times 256$ & $2,7,20,21,43$ & Wavelets \\
\hline [16] & USS & noi: 16 & $3,13,41$ & Gabor \\
\hline$[17]$ & $\begin{array}{l}\text { PARVIS [18] } \\
\text { TILDA [19] }\end{array}$ & & $\begin{array}{c}\text { PARVIS: } 2,3,14,17,20,21,22,32, \\
44,45,46 \\
\text { TILDA: } 2,3,61,20,22,37,46,47 \\
48\end{array}$ & Second-order statistics \\
\hline$[20]$ & $U$ & & $3,8,10,61,24$ & $\begin{array}{l}\text { Filters }+ \text { threshold }+ \text { erosion } \\
\text { process }+ \text { labeling algorithm }\end{array}$ \\
\hline$[21]$ & $U$ & $\begin{array}{c}\text { ndi: } 48, \text { nwi:39, } \\
256 \text { × } 256 \\
\end{array}$ & $19,26,28,31,49,50$ & GWN and morphological filters \\
\hline$[22]$ & $U$ & ndi: 56, nwi:64 & $2,7,14,15,20,21,32,43$ & MAW \\
\hline$[23]$ & $U$ & & $3,10,11,61,20,33,34,35$ & Autocorrelation \\
\hline$[24]$ & TILDA [19] & noi: $400,768 \times 512$ & $3,61,37$ & Gabor wavelets \\
\hline$[25]$ & PU & $256 \times 256$ & $3,5,7,46,54$ & WT \\
\hline$[26]$ & PU & & $\begin{array}{l}\text { Centralized, long narrow, and } \\
\text { irregular defects }\end{array}$ & Gray feature model \\
\hline $\begin{array}{l}27] \\
{[28]} \\
{[29]}\end{array}$ & $\begin{array}{l}\text { S [30] } \\
\text { PC }\end{array}$ & $\begin{array}{l}\text { S noi: } 78,256 \times 256 \\
\text { PC } 2048 \times 256 \text { or } \\
768 \times 256\end{array}$ & $\begin{array}{c}1,7,8,19,26,28,31,43,47,49 \\
50,56,57,58,59\end{array}$ & 2D GWT \\
\hline [31] & $\mathrm{PC}$ & noi: 56 & $1,3,21,60$ & Autocorrelation \\
\hline [32] & $\mathrm{P}$ & nwd: 32 & 3,61 & Wavelets \\
\hline [33] & $\mathrm{P}$ & ndi: 25, nwi:25 & $1,10,11,12,27,49,55,57$ & Gabor \\
\hline [34] & $\mathrm{PC}$ & $256 \times 256$ & $3,4,27,29,46$ & AR model and SVDD \\
\hline [35] & PU & $256 \times 256$ & $5,15,61,39,42$ & GLCM and SVDD \\
\hline$[36]$ & TILDA [19] & & $\begin{array}{l}\text { Three groups: point defect, line } \\
\text { defect, and surface defect }\end{array}$ & Regional growing PCNN \\
\hline [37] & PU & & $\begin{array}{l}\text { Horizontal, vertical, and diagonal } \\
\text { line defects }\end{array}$ & Wavelets \\
\hline [38] & PUC SD & $1920 \times 1080$ & $\begin{array}{c}\text { Horizontal, vertical, and diagonal } \\
\text { defect }\end{array}$ & Gabor and PCNN \\
\hline [39] & PUC & $320 \times 420$ & Defects not specified & SVM \\
\hline [40] & PUC & $\begin{array}{l}\text { ndi: } 40, \text { nwi: } 60 \\
\quad 400 \times 400\end{array}$ & 3,5 & DNN \\
\hline [41] & $\begin{array}{l}\text { TILDA [19] } \\
\text { Their own } \\
\text { database PC }\end{array}$ & $\begin{array}{l}\text { noi: } 50,768 \times 512 \\
\text { ndi: } 102, \text { nwi: } 102 \\
\quad 1050 \times 1050\end{array}$ & $\begin{array}{c}\text { TILDA: } 3,8,39,61 \\
\text { Own database: defects classified } \\
\text { into three groups: yarn arrangement, } \\
\text { tonal defects, and subtle defects }\end{array}$ & $\begin{array}{l}\text { Nonlocally centralized sparse } \\
\text { representation }\end{array}$ \\
\hline
\end{tabular}

$\mathrm{AR}$, auto regressive; $\mathrm{B}$, backlighting condition; $\mathrm{C}$, captured through cameras in a real prototype; DNN, deep neural network; FIR, finite impulse response; GLCM, gray-level co-occurrence matrix; GWN, Gabor wavelet network; GWT, Gabor wavelet transform; MAW, multiple adaptive wavelets; ndi, number of defect images; noi, number of images; nwi, number of images without defect; P, private (not accessible); PCNN, pulsecoupled neural network; R, resolution in width $\times$ height (8 bits per pixel); S, scanned images; SD, simulated defect; SS, simulated images; SVDD, support vector data description; SVM, support vector machine; $U$, unknown (no data given, no sample images); WT, wavelet transform.

TIF pictures (768 x 512 pixels, gray-level image 8 bits) were acquired through relocation and rotation of the textile sample. This database is difficult to access as users have to pay [4].

\section{Detection methods}

The aim of this section was to give a general overview of the most commonly used methodologies in the detection of 


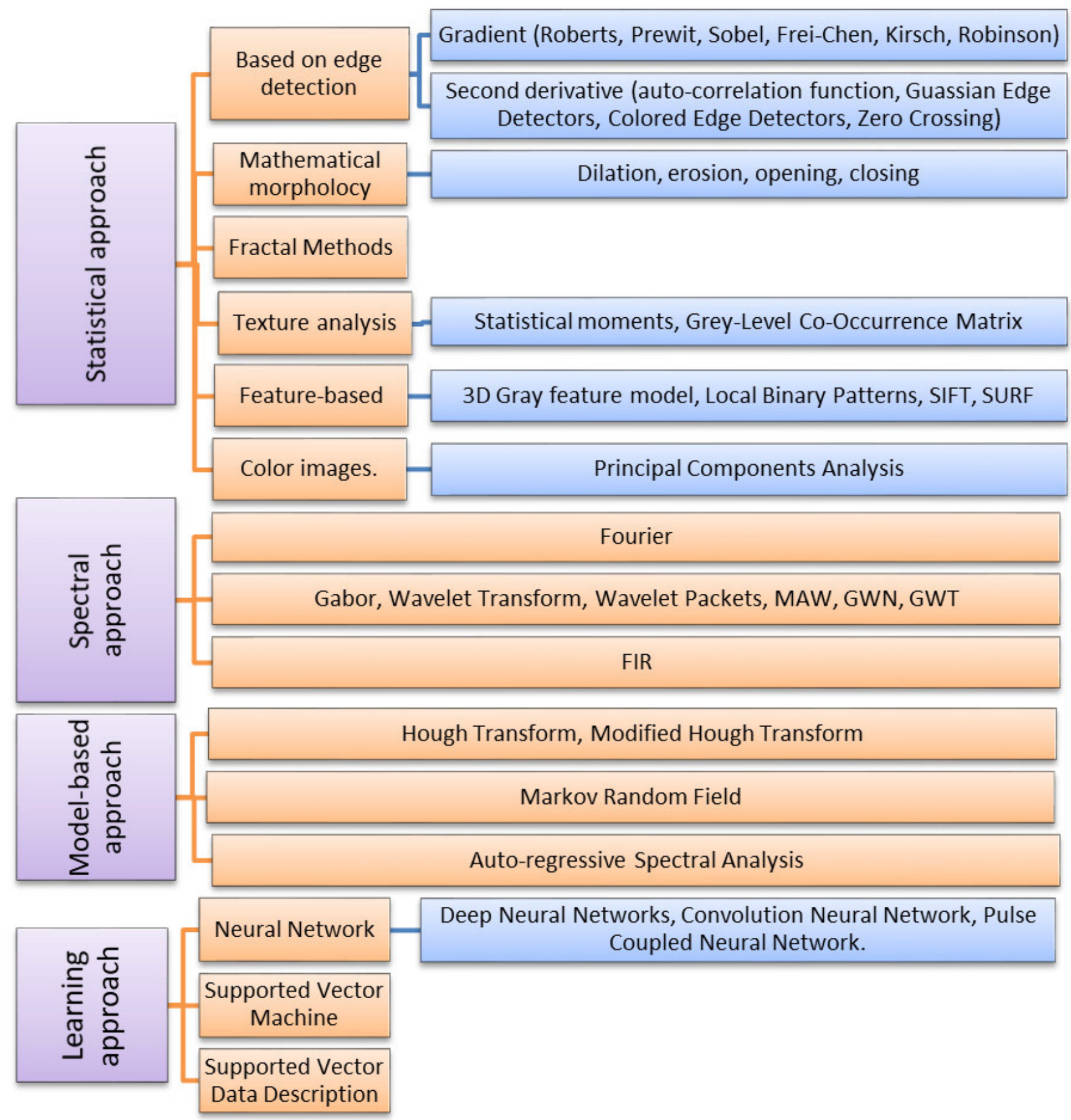

Figure 1. Classification of defect detection methods

defects. There are a number of different detection methods, and according to the bibliography, they can be divided into different groups.

Most authors divide these methods into three groups [1]: statistical, spectral, and model based, but in recent years, the learning approach has become important [4]. In this paper, fabric defect detection methods are categorized into four classes as shown in Figure 1.

The first group, statistical approach, includes a number of methods, such as those based on edge detection, which apply a border detector to the image. These techniques cover the methods based on first-order derivative edge detection or gradient and those based on second derivative and the autocorrelation function [10, 23], Gaussian edge detectors, colored edge detectors, and zero crossing, as well as methods based on mathematical morphology [56], in which the geometric aspect and the topology of the objects are the relevant parameters. The fractal method is also used in textile detection [57]. On the other hand, the analysis of textures can be carried out using statistical methods that mainly involve the statistical moments and the gray-level co-occurrence matrix (GLCM) [35]. There are also feature-based techniques, in which a pixel 
is assigned to one region according to the local features of the image in this pixel and in its immediate neighbors. This group encompasses segmentation techniques that work by gray level [26] and segmentation based on local binary patterns for defect detection in patterned and unpatterned fabrics [53]. Other methods based on features are scale-invariant feature transform (SIFT) technique [58] and other techniques based on SIFT such as speeded-up robust features (SURF) [59]. Other methods from this first group are those based on segmentation of images in color, for example, principal components analysis [60] and segmentation based on low-level features for color and texture [61].

Spectral approaches are classified in Refs. [1] and [2] such as Fourier $[62,63]$ that work in the frequency domain to characterize the defects; Gabor [60, 64] used in the analysis of the textured images: wavelet transform (WT) that offers localized information from different directions $[65,66]$ and other algorithms based on these methods such as wavelet packets [11], multiple adaptive wavelets [22], Gabor wavelet networks [21], Gabor WT [29], Mallat WT [67], and approaches based on optimized filters, for example, finite impulse response (FIR) filters [14].

The third group of methods to be discussed is model based. These presuppose that some features of the object or region are previously known: straights, circular objects, etc. These include projections and Hough transform [68] or its variants modified HT [69]. Other methods based on models include Markov random field [70] used in fabric inspection and autoregressive spectral analysis [34].

The last group of techniques is learning approaches. The defect detection can be considered as a one-class classification problem [35, 34]. Several machine-learning-based texture classification methods have been proposed using artificial neural networks (ANNs), support vector machine (SVM), and support vector data description (SVDD). Algorithms based on ANN, or simply neural network (NN), have also attracted a lot of attention in defect detection applications; ANN is expected to be widely used in future fabric defect detection systems [4]. See Ref. [7] in which NNs have been used, and [36] and [38] in which pulse-coupled NNs (PCNNs) have been used for fabric defect detection. In Ref. [71], a color channel separation using an NN, which segments the jacquard image into color channel images, was carried out. Deep NNs (DNNs) are currently popular in defect detection, for example, in Ref. [40], the authors developed a method based on deep learning to fabric defect detection. Convolution NNs (CNNs) have attracted much attention in many fields such as object detection; in Ref. [72], CNN classification in combination with SURF is used to classify dry-washed textiles such as fiber with defect or without defect. Another classifier used for fabric defect detection is SVM [39]. The one-class classifier, SVDD, is adopted as the detector in Refs. [35, 34].

\section{Use of Gabor filters for fabric defect detection}

Texture analysis methods can be used for problems related to classification, segmentation, synthesis, shape analysis, recovery of images in a database, etc. The detection method for defects used in the tests on images from the database presented in this paper is based on the use of Gabor filters. This is a spectral method based on texture analysis widely used in defect detection, including Refs. [73], [74], and others works such as $[60,75]$. Gabor filters are considered to be the most successful method, of the non-feature extraction detection schemes, for detecting fabric defects [5]. Anyway, it was not the aim of this paper to determine which is the most appropriate method for defect detection, or to carry out a comparison of methods, but rather to show an example using the database proposed here.

Gabor filters are band-pass filters created as a result of the multiplication of a Gaussian envelope function with a complex oscillation. The main advantage of introducing this Gaussian envelope is that the Gabor functions are localized in both the spatial and frequency domain. The function of Gabor in the 2D space domain can be expressed as:

$G(x, y ; \lambda, \theta, \psi, \sigma, \gamma)=\exp ^{\left(-\frac{x^{2}+\gamma^{2} y^{2}}{2 \sigma^{2}}\right)} \exp ^{i\left(2 \pi \frac{x^{\prime}}{\lambda}+\psi\right)}$

and y' arise from a movement and axis rotation given by $\theta$, in such a way that:

$x^{\prime}=x \cos \theta+y \sin \theta$.

$y^{\prime}=-x \sin \theta+y \cos \theta$

where real and imaginary components of this complex function can be calculated as:

$$
\begin{aligned}
& G(x, y ; \lambda, \theta, \psi, \sigma, \gamma)=\exp ^{\left(-\frac{x^{\prime 2}+\gamma^{2} y^{2}}{2 \sigma^{2}}\right)} \cos \left(2 \pi \frac{x^{\prime}}{\lambda}+\psi\right) \\
& G(x, y ; \lambda, \theta, \psi, \sigma, \gamma)=\exp \left(-\frac{x^{\prime 2}+\gamma^{2} y^{\prime 2}}{2 \sigma^{2}}\right) \sin \left(2 \pi \frac{x^{\prime}}{\lambda}+\psi\right)
\end{aligned}
$$

where $\lambda$ represents the wavelength, $\theta$ represents the orientation, $\psi$ is the phase offset, $\sigma$ is the typical Gaussian deviation, and $V$ is the spatial aspect ratio. A range of Gabor filters can be constructed, creating what is known as a filter bank. This is done by varying $\lambda, \theta, \psi$, and $\sigma$.

Detection using Gabor filters can be carried out in two ways $[2,28,33]$ : first, using a filter bank, and second using optimum filters. The use of filter banks reinforces the behavior of the segmentation process but generates a large quantity of data for processing [60] and the computing time needed is excessive. On the other hand, the optimum filters can counter these negative effects in specific problems. Optimum filters are used to detect a particular texture. Fewer filters are used, and therefore the filtering time is shorter; however, the choice of parameters is both crucial and complicated [64]. In Figure 2, 


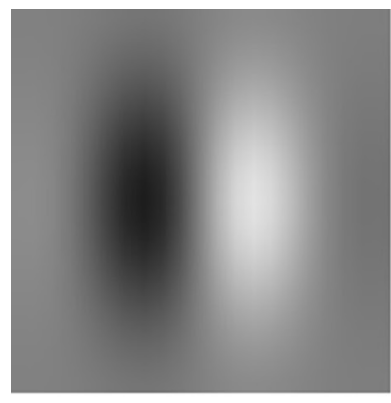

(a)

\section{$0.48-1.50 \mathrm{rad}$}

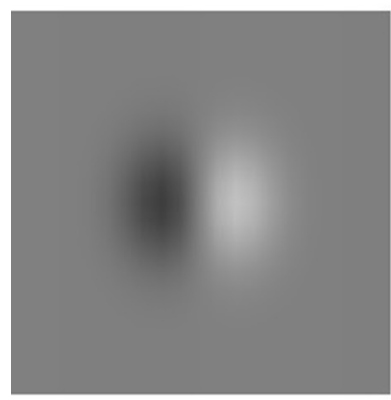

(e)

$$
0.48-1.5 \pi / 2 \mathrm{rad}
$$

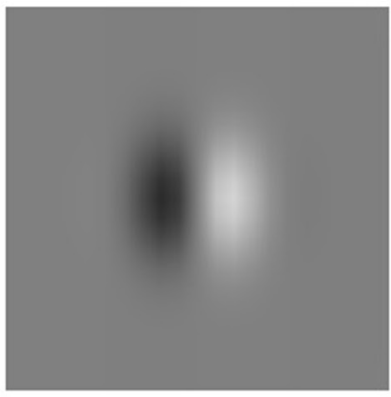

(f)

\section{$0.24-1.50 \mathrm{rad}$}

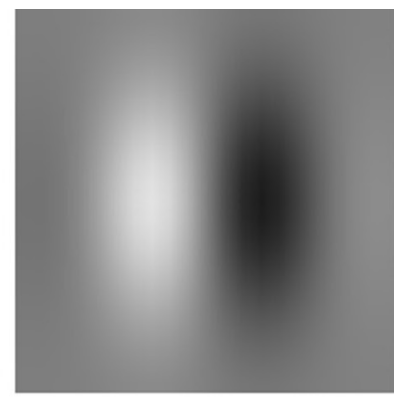

(c)

\section{$0.48-1.5 \pi \mathrm{rad}$}

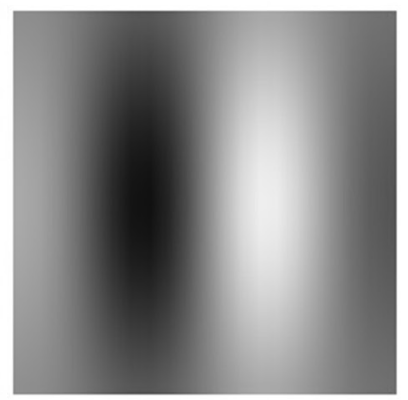

$(\mathrm{g})$

\section{$0.71-1.50 \mathrm{rad}$}

\section{$0.71-2.50 \mathrm{rad}$}

Figure 2. Images of Gabor filter kernels with different parameters $\sigma-\lambda \theta$

different images of Gabor filter kernels are shown. The values of the parameters used are indicated below every image in the following order $\sigma-\lambda \theta$.

The two components of the Gabor filter (real and imaginary) may form a complex number or may be used individually. According to Mak et al. [28], the filter based on the imaginary part is to attenuate the areas without defect or highlight the areas with defect in the image. The value of $\theta$ must be selected with great care to eliminate the base texture, and these parameters depend on the type of textile. The Gabor filter based on the real part is used to detect the contours of defect objects. In the literature, applications of only one type of filter can be found, such as in Ref. [33] in which the authors use an initial training stage to determine the filter parameters with a defect-free image and design an uneven Gabor filter to detect defects that have the same textural background as the sample.

\section{Our database}

\subsection{Capture system}

The system used to capture images is composed of a linear GigaEthernet camera, LED linear lighting, an encoder for the synchronization, a PC for processing and detection and a tablet for labeling, as shown in Figure 3 . The camera has

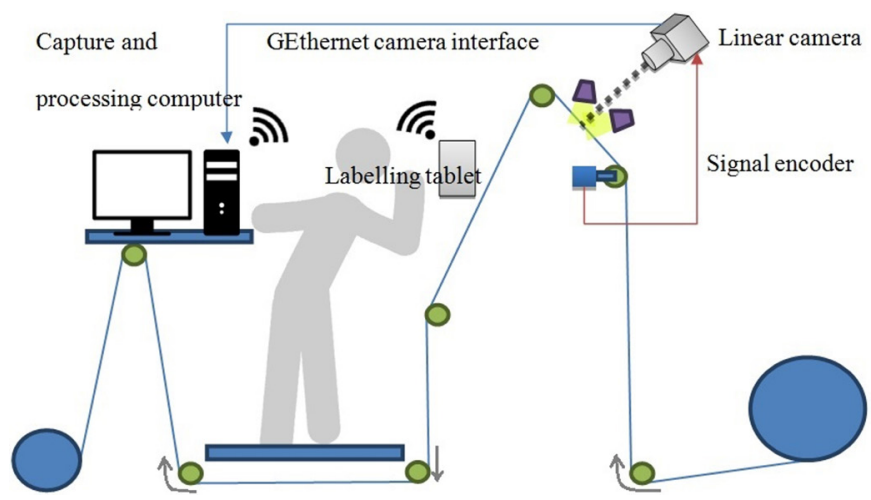

Figure 3. Plan of the capture system

4096-line capture pixels which, with a resolution of four pixels per millimeter, cover the same half of the fabric that is being inspected by the operator who has to manage the system and the tablet for labeling.

The acquisition system, which also carries out processing and labeling functions on the images, is done using a PC in which the same capture software has a server incorporated which receives the defect labeling data via sockets. The labeling is carried out using an Android application implemented in a tablet in which the operator, after introducing the data from the piece being manufactured, manages to mark all the defects found in the fabric (Figure 4). The labeling is sent to the server installed 


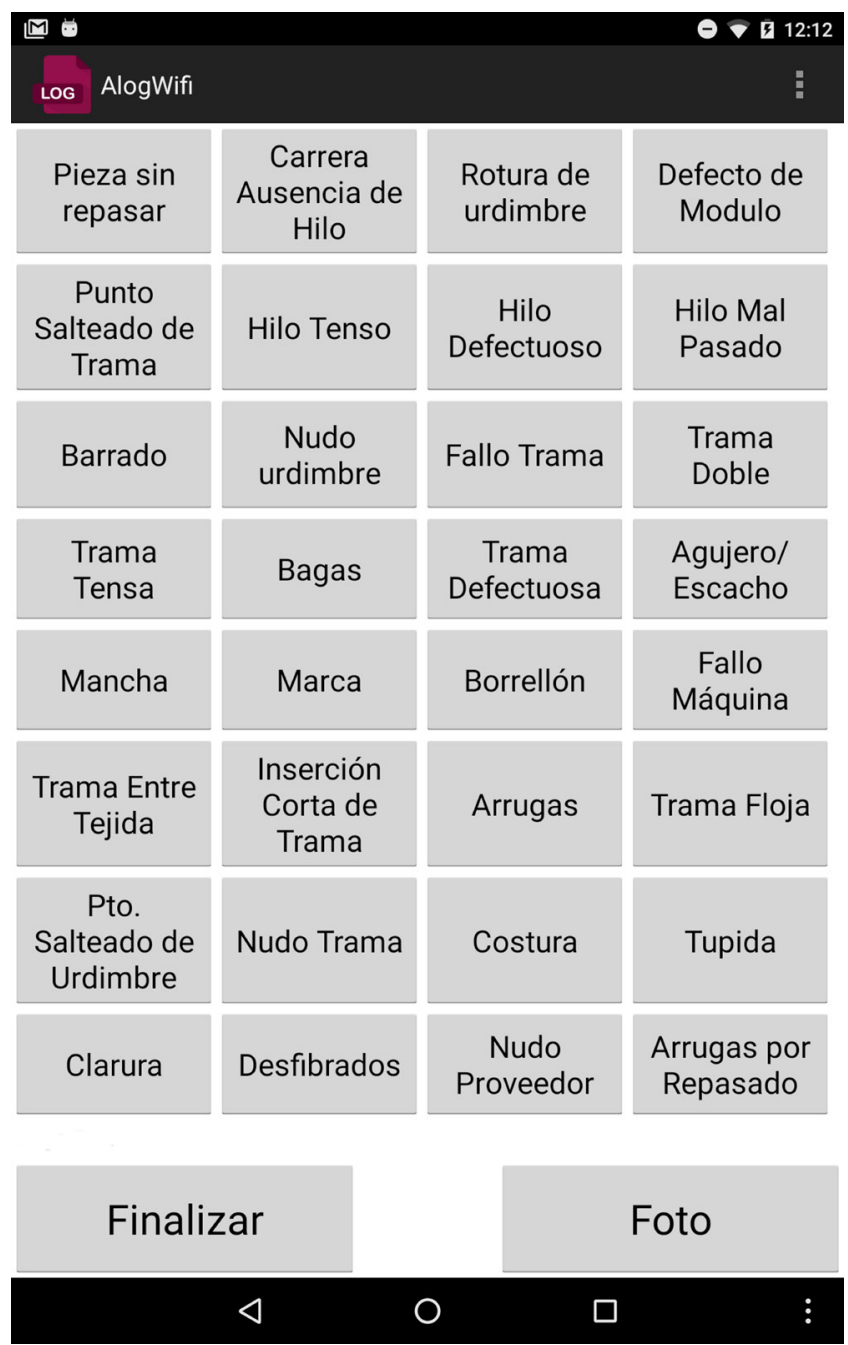

Figure 4. Android application to label defects

in the capture software which stores these data alongside the images captured. As the process to detain the production process when a defect is detected, and then label this defect, is carried out by the operator, the process is a manual one. The defect is then sought manually from among a small number of stored images. In this way, real, trustworthy information is obtained on the defects found on the fabric, and the results produced by the detection algorithms can be compared using this information.

\subsection{The database}

The database consists of 245 images of $4096 \times 256$ pixels captured by the system of seven different fabric structures. The fabrics in the database are mainly plain. There are 140 defectfree images in the database, 20 of every type of fabric. The other images, of which there are 105, contain different types of fabric defects which commonly appear in the textile industry (we have 12 types of defect). The large size of the images

Table 3. Values of the parameters selected in the detection method used

\begin{tabular}{|c|c|c|c|c|}
\hline & $\boldsymbol{\theta}$ & $\boldsymbol{\sigma}$ & $\boldsymbol{\lambda}$ & $\boldsymbol{\Psi}$ \\
\hline Test 1 & \multirow{2}{*}{ Odd filter: $0, \boldsymbol{\pi} / \mathbf{2}, \boldsymbol{\pi}, \mathbf{3} \boldsymbol{\pi} / \mathbf{2}$} & 0.24 & 2.5 & 0 \\
\cline { 1 - 3 } Test 2 & & 0.29 & 2.5 & 0 \\
\hline
\end{tabular}

\section{ImageNumber_DefectCode_FabricCode.png}

ImageNumber_DefectCode_FabricCode_mask.png

Figure 5. Example of how the images of the database are denominated

allows users to work with different window sizes, increasing the number of samples.

These images can be accessed on the Internet (www.aitex.es/ afid). The images have been named as shown in Figure 5.

An example of the 12 defects and at least one example of each of the seven different fabric structures are shown in Figure 6. Each figure shows a region of interest (ROI) of $256 \times 256$ pixels, in place of $4096 \times 256$ original image, so that the structure and defects in the textile can be seen in detail.

The database available on the Internet also contains the segmentation mask of all the images with defects in such a way that the white pixels indicate the defective area, and the rest of the pixels are black. The masks have been marked and created by hand from the original image. As the defect is sometimes difficult to localize in the original image of 4096 pixels in length, these masks will help other researchers localize the defects in this set of images, as the original image can be compared with its respective mask. Four examples are shown in Figure 7.

\section{Experimental results}

The processing of the images for the detection of defects consisted of an initial preprocessing before the application of a Gabor filter bank. A bank of filters was used in which an uneven filter was applied for different values of $\theta(0, \pi / 2,3 \pi / 2)$. That is to say, Gabor was applied four times, only varying the values of $\theta$, while the rest of the values used in the filter, sigma, lambda, and psi, were always fixed in a particular test. When using Gabor, an important step is to determine the most appropriate parameters. After analyzing thousands of fabric images, the values that offer the best results for defect detection have been identified, and these values are used in this paper. Table 3 summarizes the values used in the tests presented in this paper, only the values of $\sigma$ are changed, while the values for $\theta, \lambda$, and $\psi$ are fixed. After the application of each Gabor filter, the image is thresholded and the shape of the defect is reflected in the obtained image. Each shape of defects is added to a final image, that is, the complete defect can be obtained through image addition. After that, a morphology filter is applied to the complete defect image. Finally, the contours corresponding to the defects are sought and these are marked as a result of the detection.

Figure 8 shows the selection of the results obtained with the defect detection method used. The images show a ROI of $256 \times 256$ pixels in which the defect is located. In the final stage of the detection process, the defect detected is framed in the original image with a rectangle. As an example, four different textile samples containing four different defects are shown. 


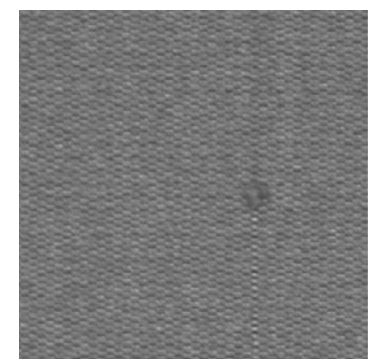

(a) 0089_002_01.png

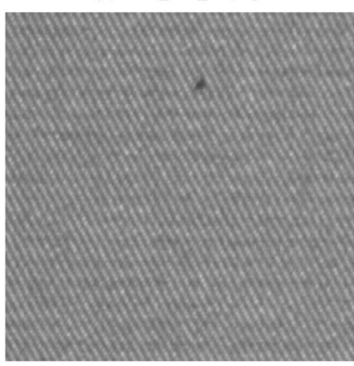

(e) 0057_019_06.png

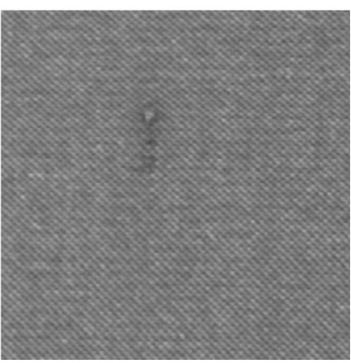

(i) 0094_027_05.pns

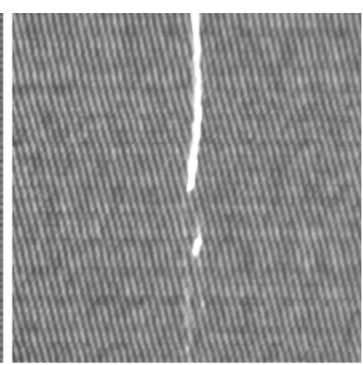

(b) 0015 _006_02.png

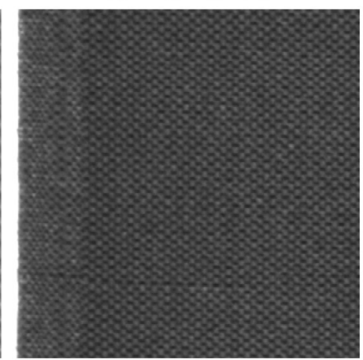

(f) 0064_022_00.png

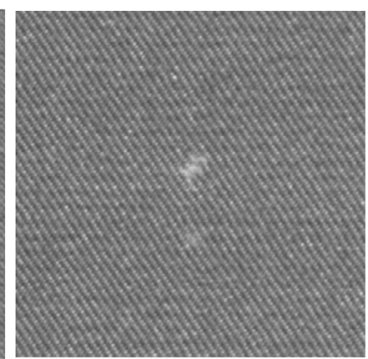

(j) 0083_029_04.png

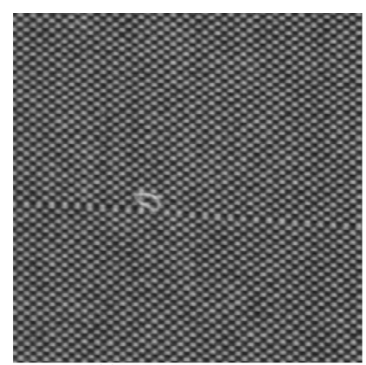

(c) 0075_010_03.png

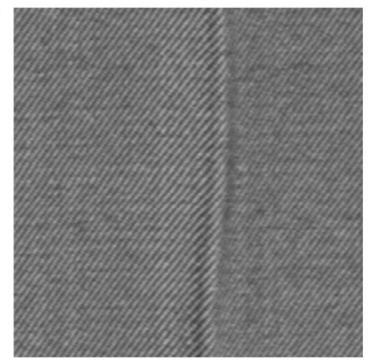

(g) 0069_023_02.png

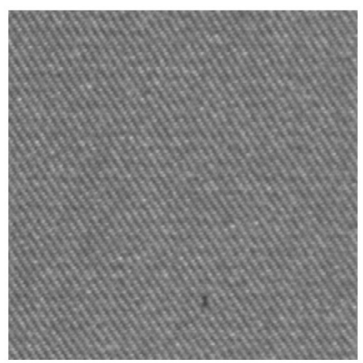

(k) 0084_030_04.png

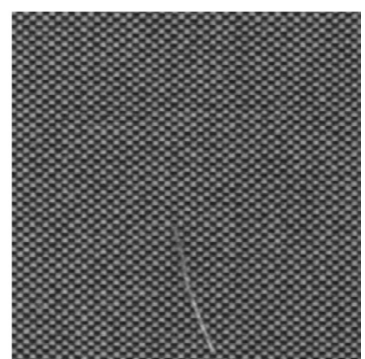

(d) 0019_016_03.png

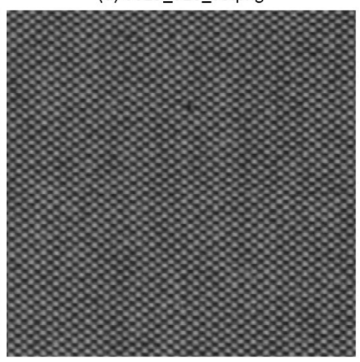

(h) 0107_025_03.png

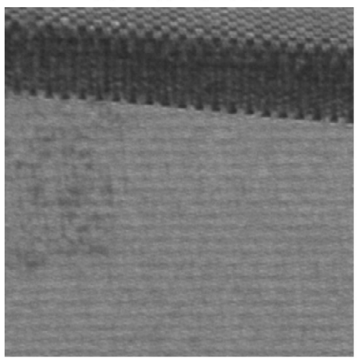

(I) 0080_036_01.png

Figure 6. ROI of $256 \times 256$ pixels from original examples $4096 \times 256$ of defective fabrics, with the names used in the database. (a) broken end, (b) broken yarn, (c) broken pick, (d) weft curling, (e) fuzzy ball, (f) cut selvage, (g) crease, (h) warp ball, (i) knot, (j) contamination, (k) nep, and (I) weft craft. ROI, region of interest
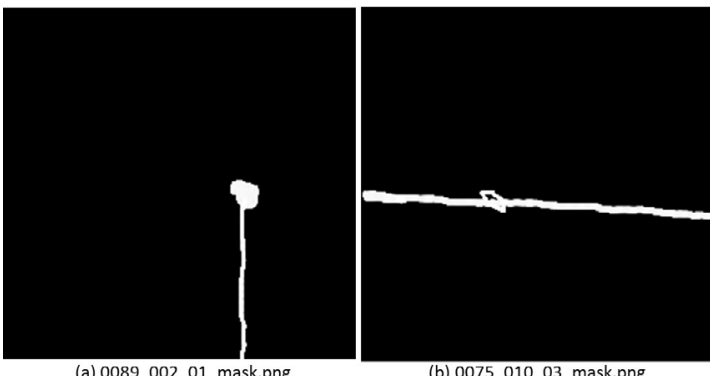

(b) 0075_010_03_mask.png

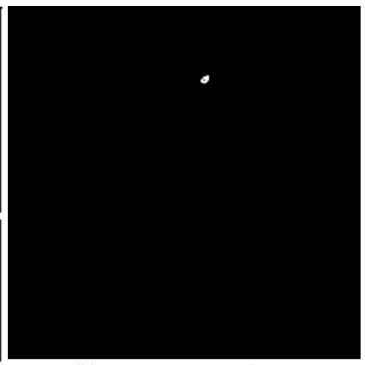

(b) 0057_019_06_mask.png

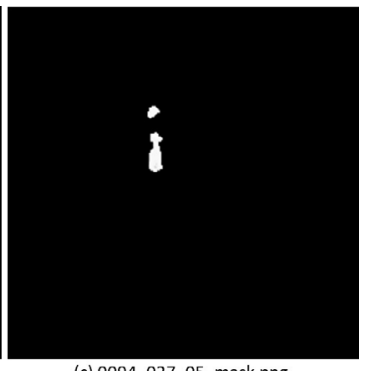

(c) 0094_027_05_mask.png

Figure 7. ROI of $256 \times 256$ pixels of the image mask of Figure 6 (a), (c), (e), (i). ROI, region of interest

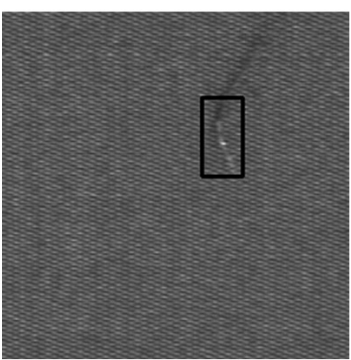

(a) 0003_002_00.png

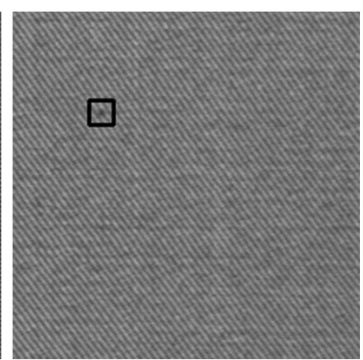

(b) 0034_019_02.png

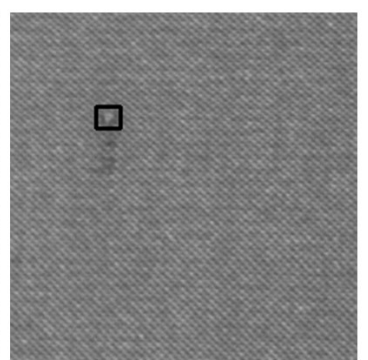

(c) 0094_027_05.png

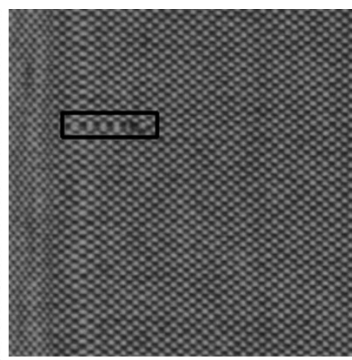

(d) 0104_022_03.png

Figure 8. Examples of fabric defect detection. (a) broken end, (b) fuzzy ball, (c) knot, (d) cut selvage

The performance of the defect detection scheme used can be determined in different ways. Some authors state that there are two ways to measure the accuracy of detection [2]: (1) detection success rate (DSR) or (2) sensitivity and specificity.
DSR, also known as detection accuracy, can be defined as the number of samples correctly detected divided by the total number of samples, defective and defect free (6). The DSR is a metric used by different researchers, see Refs. [2] and [7]. 
Other relationships used are detection rate (DR) (7) and false alarm rate (FAR) (8).

$$
\mathrm{DSR}=\frac{\text { number } \text { of samples correctly detected }}{\text { total number of samples }} \times 100
$$

$$
D R=\frac{\text { number of defective samples correctly detected }}{\text { total number of defective samples }} \times 100
$$

$F A R=\frac{\text { Number of defect free samples detected as defective }}{\text { Total number of defect free samples }} \times 100$

where the accuracy of the method using metrics such as "specificity" is defined as correct detection of defect-free samples (10), and "sensitivity" or correct detection of defective samples (9) is evaluated. To calculate these values, the definitions of true positive (TP), false positive (FP), false negative (FN), and true negative (TN) are necessary (Table 4).

$$
\text { Sensitivity }=\frac{\mathrm{TP}}{\mathrm{TP}+\mathrm{FN}} \times 100
$$

$$
\text { Specificity }=\frac{T N}{T N+F P} \times 100
$$

$$
\text { Accuracy }=D S R=\frac{T N+T P}{T N+F P+F N+T P} \times 100
$$

Based on the metrics mentioned above, the results of the evaluation of the method used in this paper are now presented. The parameters for these tests are previously summarized in Table 3. It is worth highlighting that the number of samples for each defect is not the same. This is so because the data were collected from a real installation where the defects appear at different rates. During the months in which the data were being collected, there were defects that were never registered or only occasionally registered, which also helps to focus the defect detection, avoiding a situation where methods are being developed that only detect a particular type of defect, when this type of defect hardly ever occurs during production. However, this distribution may be different in other production plants in which different threads and weaves are used.

The testing results were quantified using sensitivity to show how accurately defective samples were classified, specificity to show how accurately defect-free samples were classified, false alarm, and accuracy as the correct classification rate of all samples (Table 5). As can be seen, the results improved in the second test.

If the parameters are more "conservative" (test 1), the DR is lower but the FN and FA are almost insignificant, 4/140. However, if the parameters are modified (test 2), increasing the value of $\sigma$, the DR increases but more false defects are obtained. In Ref. [2], an exhaustive review about automated fabric defect detection methods is given, with many of the works mentioned obtaining similar results to those shown here. Although the values obtained are not very high, the objective of the paper was not the detection method as already mentioned. In addition, it must be taken into account that they are real defects, with greater difficulty of detection to those presented in other papers such as Ref. [32] where the authors accomplished $91 \%$ and $100 \%$ DSRs for 16 images of holes and 16 images of oil stain, respectively, using ANNs.

The parameters can be varied and different values can be used according to the textile type; thus, false defects and detection in general would be improved; however, this is outside the scope of this present work.

\section{Conclusions}

Defect detection in industrial textile manufacture is a basic need for which efficient economical solutions must be sought. In recent years, a number of different methods have been developed to detect these defects, some of which have been successful to a greater or lesser degree, although it has generally been impossible to verify the exact degree of success achieved. This is due to the use of a collection of private images, which are not publicly available, and thus they cannot be used to verify any proposed results or make comparisons between methods. In this paper, a set of annotated images and their corresponding segmentations is presented, with and without defects, of different types of plain textiles. This set of images is now available on the Internet. The images were obtained from

Table 4. Definitions of TP, FP, TN, and FN in defect detection

\begin{tabular}{|c|c|c|}
\hline & Image defective & $\begin{array}{c}\text { Image defect } \\
\text { free }\end{array}$ \\
\hline $\begin{array}{c}\text { Detected as } \\
\text { defective }\end{array}$ & TP & FP \\
\hline $\begin{array}{c}\text { Detected as } \\
\text { defect free }\end{array}$ & FN & TN \\
\hline
\end{tabular}

$\mathrm{FN}$, false negative; FP, false positive; TN, true negative TP, true positive.

Table 5. Performance analysis of the method used

\begin{tabular}{|c|c|c|c|c|}
\hline & Sensitivity or DR (\%) & Specificity (\%) & FAR (\%) & DSR (\%) \\
\hline Test 1 & $82 / 105=78.10$ & TN/140=136/140=97.14 & 2.90 & 88.98 \\
\hline Test 2 & $91 / 105=86.67$ & TN/140=124/140=88.57 & 11.4 & 87.76 \\
\hline
\end{tabular}

$\mathrm{DR}$, detection rate; DSR, detection success rate; FAR, false alarm rate; TN, true negative. 
a real production plant and were generated from a wide range of defects, and moreover, came from seven different textile types with a range of different textures. The capture system was complemented with a detection system based on Gabor filters which proved the reliability of this technology for realtime deployment in industry. This technology will be extremely important in the coming Factory 4.0 trend. Regarding future lines of research in this field, the aim is to install more machines in other factories, so that the database of images with and without defects can continue to grow. At the same time, the objective is to develop mechanism to handle and manage this large quantity of data. Moreover, the volume of data that hopefully will eventually be achieved will mean that Big Data techniques can be used for the detection and classification of defects.

\section{Acknowledgement}

The authors thank for the financial support provided by IVACE (Institut Valencià de Competitivitat Empresarial, Spain) and FEDER (Fondo Europeo de Desarrollo Regional, Europe), throughout the projects: AUTOVIMOTION and INTELITEX.

\section{References}

[1] Kumar, A. (2008). Computer-vision-based fabric defect detection: a survey. IEEE Transactions on Industrial Electronics, 55(1), 348-363.

[2] Ngan, H. Y., Pang, G. K., Yung, N. H. (2011). Automated fabric defect detection - a review. Image and Vision Computing, 29(7), 442-458.

[3] Habib, T., Faisal, R., Rokonuzzaman, M., Ahmed, F. (2014). Automated fabric defect inspection: a survey of classifiers. International Journal in Foundations of Computer Science \& Technology (IJFCST), 4(1), 17-25.

[4] Hanbay, K., Talu, M., Özguven, Ö. (2016). Fabric defect detection systems and methods. A systematic literature review. Optik, 127, 11960-11973.

[5] Goyal, A. (2018). Automation in fabric inspection, in Automation in Garment Manufacturing, Woodhead Publishing, 75-107.

[6] Hillel, A. B., Lerner, R., Levi, D., Raz, G. (2014). Recent progress in road and lane detection: a survey. Machine Vision and Applications, 25, 727-745.

[7] Rebhi, A., Benmhammed, I., Abid, S., Fnaiech, F. (2015). Fabric defect detection using local homogeneity analysis and neural network. Journal of Photonics, 2015, 2015.

[8] ASTM. (2016). Standard Terminology Relating to Fabric Defects. Designation: D 3990 - 12," ASTM, West Conshohocken.

[9] Ahmed, A. (2016). A catalogue of visual textile defects. TS3B.

[10] Sari-Sarraf, H., Goddard, J. S. (1999). Vision system for on-loom fabric inspection. IEEE Transactions on Industry Applications, 35(6), 1252-1259.

[11] Kumar, A., Pang, G. (2001). Identification of surface defects in textured materials using wavelet packets, in Industry Applications Conference, 2001. Thirty-Sixth IAS Annual Meeting. Conference Record of the 2001 IEEE.
[12] Wen, C.-Y., Chiu, S.-H., Hsu, W.-S., Hsu, G.-H. (2001). Defect segmentation of texture images with wavelet transform and co-occurrence matrix. Textile Research Journal, 71(8), 743-749.

[13] Zeng, P., Hirata, T. (2002). On-loom fabric inspection using multi-scale differentiation filtering, in Industry Applications Conference, 37th IAS Annual Meeting. Conference Record of the, Pittsburgh, PA, USA.

[14] Kumar, A., Pang, G. (2002). Defect detection in textured materials using optimized filters. IEEE Transactions on Systems, Man, and Cybernetics, 32(5), 553-570.

[15] Yang, X. Z., Pang, G., Yung, N. (2002). Discriminative fabric defect detection using adaptive wavelets. Optical Engineering, 41(12), 3116-3126.

[16] Shu, Y., Tan, Z. (2004). Fabric defects automatic detection using Gabor filters, in Intelligent Control and Automation, 2004. WCICA 2004. Fifth World Congress on, 2004.

[17] Murino, V., Bicego, M., Rossi, I. A. (2004). Statistical classification of raw textile defects, in Pattern Recognition, 2004. ICPR 2004. Proceedings of the 17th International Conference on, 2004.

[18] PARVIS, "PARVIS," provided by PARVIS srl, [Online]. Web site: $h t t p: / / w w w . p a r v i s . i t /$.

[19] Technische Universitt Hamburg-Harburg. (1995). TILDA Textile Texture-Database. [Online]. Web site: http:// Imb.informatik.uni-freiburg.de/resources/datasets/tilda. en.html.

[20] Cho, C.-S., Chung, B.-M., Park, M.-J. (2005). "Development of real-time vision-based fabric inspection system. IEEE Transactions on Industrial Electronics, 52(4), 1073-1079.

[21] Mak, K. L., Peng, P., Lau, H. Y. (2005). Optimal morphological filter design for fabric defect detection, in Industrial Technology, 2005. ICIT 2005. IEEE International Conference on, Hong Kong.

[22] Yang, X., Pang, G., Yung, N. (2005). Robust fabric defect detection and classification using multiple adaptive wavelets. IEEE Proceedings - Vision, Image and Signal Processing, 152(6), 715-723.

[23] Zhu, S. W., Hao, H. Y., Li, P. Y., Shi, M. H., Qi, H. (2007). Fabric defects segmentation approach based on texture primitive, in Machine Learning and Cybernetics, 2007 International Conference on, 2007.

[24] Basturk, A., Ketencioglu, H., Yugnak, Z., Yuksel, M. E. (2007). Inspection of defects in fabrics using Gabor wavelets and principle component analysis, in Signal Processing and Its Applications, International Symposium on (ISSPA), Sharjah.

[25] Liu, S.-G., Qu, P.-G. (2008). Inspection of fabric defects based on wavelet analysis and BP neural network, in Wavelet Analysis and Pattern Recognition, 2008. ICWAPR '08. International Conference on, Hong Kong.

[26] Liu, Z. (2009). Computer testing method of defect feature of fabric, in Test and Measurement, 2009. ICTM '09. International Conference on.

[27] Mak, K., Peng, P., Lau, H. (2005). A real-time computer vision system for detecting defects in textile fabrics, in International Conference on Industrial Technology, HongKong.

[28]Mak, K. L., Peng, P., (2008). An automated inspection system for textile fabrics based on Gabor filters. Robotics and Computer-Integrated Manufacturing, 24(3), 359369. 
[29] Mak, K., Peng, P., Yiu, K. (2009). Fabric defect detection using morphological filters. Image and Vision Computing, 27(10), 1585-1592.

[30] Graniteville Company. (1975). Manual of standard fabric defects in the textile industry.

[31] Sheng-Wang, L., Li-Wei, G., Chun-Hua, L. (2009). Fabric defects detecting and rank scoring based on Fisher criterion discrimination, in Machine Learning and Cybernetics, 2009 International Conference on, 2009.

[32] Yin, Y., Lu, W. B., Zhang, K., Jing, L. (2009). Textile flaw detection and classification by wavelet reconstruction and BP neural network, in Intelligent Systems, 2009. GCIS '09. WRI Global Congress on, Xiamen.

[33] Fan, L., Jiang, G. (2010). Optimized Gabor filter parameters for uniform texture flaw detection, in Intelligent Systems and Knowledge Engineering (ISKE), 2010 International Conference on, Hangzhou.

[34]Bu, H. G., Huang, X. B., Wang, J., Chen, X. (2010). Detection of fabric defects by auto-regressive spectral analyis and support vector data description. Textile Research Journal, 89(7), 579-589.

[35] Mingde, B., Zhigang, S., Yesong, L. (2012). Textural fabric defect detection using adaptative quantized gray-level cooccurrence matrix and support vector description data. Information Technology Journal, 11, 673-685.

[36] Si, X., Zheng, H., Hu, X. (2012). Fabric defect detection based on regional growing PCNN. Journal of Multimedia, 7(5), p. 372.

[37] Karlekar, V. V., Biradar, M. S., Bhangale, K. B. (2015). Fabric defect detection using wavelet filter, in Computing Communication Control and Automation (ICCUBEA), 2015 International Conference on, Pune.

[38] Li, Y., Zhang, C. (2016). Automated vision system for fabric defect inspection using Gabor filteres and PCNN. SpringerPlus, 5(1).

[39] Kumbhar, P., Mathpati, T., Kamaraddi, R., Kshirsagar, N. (2016). Textile fabric defects detection and sorting using image processing. International Journal for Research Emerging Science and Technology, 3(3), 19-24.

[40] Seker, A., Peker, K., Yüksek, A. D. E. (2016). Fabric defect detection using deep learning, in 24th Signal Processing and Communication Application Conference (SIU), Zonguldak.

[41] Tong, L., Wong, W., Kwong, C. (2017). Fabric defect detection for apparel industry: a nonlocal sparse representation approach. IEEE Access, 5, 5947-5964.

[42] The Berkeley Segmentation Dataset and Benchmark. (2013). [Online]. Web site: https://www2.eecs.berkeley. edu/Research/Projects/CS/vision/grouping/resources. html.

[43] Arbelaez, P., Maire, M., Fowlkes, C., Malik, J. (2011). Contour detection and hierarchical image segmentation. IEEE Transactions on Pattern Analysis and Machine Intelligence, 33(5), 898-916.

[44] Jackowski, K., Cyganek, B. (2015). A learning-based colour image segmentation with extended and compact structural tensor feature representation. Pattern Analysis and Applications, 20, 1-14.

[45] Dong, Y., Ma, J. (2012). Bayesian texture classification based on contourlet transform and BYY harmony learning of Poisson mixtures. IEEE Transactions on Image Processing, 21(3), 909-918.
[46] Selvan, S., Ramakrishnan, S. (2007). SVD-based modeling for image texture classification using wavelet transformation. IEEE Transactions on Image Processing, 16(11), 2688-2696.

[47] Li, L., Tong, C. S., Choy, S. K. (2010). Texture classification using refined histogram. IEEE Transactions on Image Processing, 19(5), 1371-1378.

[48] Brodatz, P. (1996). Textures: a photographic album for artists and designers, Dover, New York. Web site: http:// www.ux.uis.no/ tranden/brodatz.html.

[49] MIT MediaLab. (1995). VisTex texture database. [Online]. Web site: http://vismod.media.mit.edu/vismod/imagery/ VisionTexture/vistex.html.

[50] Xie, X., Mirmehdi, M. (2007). TEXEMS: texture exemplars for defect detection on random textured surfaces. IEEE Transactions on Pattern Analysis and Machine Intelligence, 29(8), 1454-1464.

[51] Fritz, M., Hayman, E., Caputo, B., Eklundh, J.-O. (2006). Kth-tips database. [Online]. Web site: http://www.nada.kth. se/cvap/databases/kth-tips/.

[52] Dana, K., Van-Ginneken, B., Nayar, S., Koenderink, J. (1999). Reflectance and texture of real world surfaces. ACM Transactions on Graphics (TOG), 18(1), 1-34.

[53] Tajeripour, F., Kabier, E., Sheikhi, A. (2008). Fabric defect detection using modified local binary patterns. EURASIP Journal on Advances in Signal Processing, 2008, 60.

[54] Ngan, H. Y., Pang, G. K., Yung, S., Ng, M. K. (2005). Wavelet based methods on patterned fabric defect detection. Pattern Recognition, 38(4), 559-576.

[55] Ngan, H. Y., Pang, G. (2009). Regularity analysis for patterned texture inspection. IEEE Transactions on Automation Science and Engineering, 6(1), 131-144.

[56] Tiwari, V., Sharma, G. (2015). Automatic fabric fault detection using morphological operations on bit plane. IJCSNS International Journal of Computer Science and Network Security, 5(10), 30-35.

[57] Conci, A., Proença, C. B. (1998). A fractal image analysis system for fabric inspection based on a box-counting method. Computer Networks and Systems, 30(20-21), 1887-1895.

[58] Huang, F. C., Huang, S. Y., Ker, J. W., Chen, Y. C. (2012). High-performance SIFT hardware accelerator of real-time image feature extraction. IEEE Transactions on Circuits and Systems for Video Technology, 22(3), 340-351.

[59] Bay, H., Ess, A., Tuytelaars, T., Van Gool, L. (2008). Sepeeded-up robust features (SURF). Computer Vision and Image Understanding, 110(3), 346-359.

[60] Bissi, L., Baruffa, G., Placid, P., Ricci, E., Scorzon, A., Valigi, P. (2013). Automated defect detection in uniform and structured fabrics using Gabor filters and PCA. Journal of Visual Communication and Image Representation, 24(7), 1047-3203.

[61] Chen, J., Pappas, T. N., Mojsilovic, A., Rogowitz, B. E. (2005). Adaptive perceptual color-texture image segmentation, in Image Processing, IEEE Transactions on, 2005.

[62] Chan, C.-H., Pang, G. K. H. (2000). Fabric defect detection by Fourier analysis. IEEE Transactions on Industry Applications, 35(2), 1267-1276.

[63] Malek, A. S., Drean, J.-Y., Bigue, L., Osselin, J.-F. (2013). Optimization of automated online fabric inspection by fast Fourier Transform (FTT) and cross-correlation. Textile Research Journal, 83(3), 256-268. 
[64] Guang-Hua, H. (2014). Optimal ring Gabor filter design for texture defect detection using a simulated annealing algorithm, in Information Science, Electronics and Electrical Engineering (ISEEE), International Conference on, Sapporo, 2014.

[65] Han, Y., Shi, P. (2007). An adaptive level-selecting wavelet transform for texture defect detection. Image and Vision Computing, 25(8), 1239-1248.

[66] Guan, S., Gao, Z. (2014). Fabric defect image segmentation based on the visual attention mechanism of the wavelet domain. Textile Research Journal, 84(10), 1018-1033.

[67] Mallat, S. (1998). A wavelet tour of signal processing. Academic Press.

[68] Tsai, D.-M., Hsieh, C.-Y. (1999). Automated surface inspection for directional textures. Image and Vision Computing, 18(1), 49-62.

[69] Choi, K.-J., Lee, Y.-H., Moon, J.-W., Park, C. K., Harashima, F. (2007). Development of an automatic stencil inspection system using modified Hough transform and fuzzy logic. IEEE Transactions on Industrial Electronics, 54(1), 604611.

[70] Ozdemir, S., Ercil, A. (1996). Markov random fields and Karhunen-Loeve transforms for defect inspection of textile products, in Emerging Technologies and Factory Automation, 1996. EFTA '96. Proceedings, 1996 IEEE Conference on, 1996.
[71] Yu, X., Hu, J., Baciu, G. (2005). Defect detection of jacquard fabrics using multiple color-channel analysis. Research Journal of Textile and Apparel, 9(1), 21-29.

[72] Siegmund, D., Samartzidis, T., Fu, B., Braun, A., Kuijper, A. (2017). Fiber defect detection of inhomogeneous voluminous textiles, in Pattern Recognition, Springer International Publishing, Huatulco, Mexico, 278-287.

[73] Turner, M. R. (1986). Texture discrimination by Gabor functions. Biological Cybernetics, 55(2-3), 71-82.

[74] Clark, M., Bovik, A., Geisler, W. (1987). Texture segmentation using Gabor modulation/demodulation. Pattern Recognition Letters, 6(4), 261-267.

[75] Yanbei, L., Zhitao, X., Jun, W., Fang, Z. (2011). Fabric defect detection method based on optimal Gabor filter bank. International Journal of Digital Content Technology and its Applications, 5(11). 